Revisión

\title{
MICROORGANISMOS ANTAGONISTAS QUE INHIBEN EL DESARROLLO DE PATÓGENOS EN POST-COSECHA DE LIMONES (Citrus limon L.)
}

Review

\section{ANTAGONIST MICROORGANISMS THAT INHIBIT THE DEVELOPMENT OF POST-HARVEST PATHOGENS IN LEMONS (Citrus limon L.)}

\author{
Fabiola Soto C. ${ }^{1 *}$, Claudia Tramón P. ${ }^{1}$, Pedro Aqueveque M. ${ }^{1}$, y Johannes de Bruijn ${ }^{1 *}$
}

${ }^{1}$ Facultad de Ingeniería Agrícola, Departamento de Agroindustrias, Universidad de Concepción, Casilla 537, Chillán, Chile.

*Autores para correspondencia E-mail: fabisoto@udec.cl; jdebruij@udec.cl

\section{RESUMEN}

La producción de limones (Citrus limon L.) frescos o secos en Chile es de gran importancia económica. Desde la cosecha del fruto hasta su consumo, pasa un tiempo considerable, en el cual, el fruto contaminado de manera natural comienza un proceso de deterioro causado principalmente por microorganismos patógenos como Geotrichum citri-aurantii, Penicillium digitatum, Penicillium italicum, y Phyllosticta citricarpa. El objetivo de este estudio fue realizar una revisión de las investigaciones recientes relacionadas con el uso de microorganismos o sus derivados para el control de patógenos. Los resultados indican que se puede inhibir el desarrollo biológico de patógenos en limón mediante el uso de microorganismos beneficiosos o antagónicos, como Bacillus amyloliquefaciens, Bacillus subtilis, Paenibacillus polymyxa, Pantoea agglomerans, Pseudomonas syringae, Candida membranifaciens, Candida utilis, Cryptococcus laurentii, Debaryomyces hansenii, Kloeckera apiculata, Metschnikowia spp., Pichia guilliermondii, Pichia membranifaciens, Rhodosporidium paludigenum, Saccharomyces cerevisiae, Aureobasidium pullulans, Nodulisporium spp., y Trichoderma spp. Finalmente, se puede concluir que el biocontrol es una técnica prometedora para disminuir pérdidas en la post-cosecha de limones.

Palabras clave: limón, cítricos, antagonista, biocontrol, patógenos post-cosecha.

\section{ABSTRACT}

The production of fresh or dried lemons (Citrus limon L.) in Chile is of great economic importance. There is a long period of time from harvest to consumption in which naturally contaminated fruit starts to decay due to the action of pathogenic microorganisms, such as Geotrichum citri-aurantii, Penicillium digitatum, Penicillium italicum, and Phyllosticta citricarpa. The aim of this study was to review recent papers related to the use of microorganisms or their derivatives for the control of pathogens. The results indicate that it is possible to inhibit the biological development of pathogens in lemon by the use of beneficial or antagonistic microorganisms, such as Bacillus amyloliquefaciens, Bacillus subtilis, Paenibacillus polymyxa, Pantoea agglomerans, Pseudomonas syringae, Candida membranifaciens, Candida utilis, Cryptococcus laurentii, Debaryomyces hansenii, Kloeckera apiculata, Metschnikowia spp., Pichia guilliermondii, Pichia membranifaciens, Rhodosporidium paludigenum,

Recibido: 14 noviembre 2017. $\quad$ Aceptado: 14 marzo 2018. 
Saccharomyces cerevisiae, Aureobasidium pullulans, Nodulisporium spp., and Trichoderma spp. Finally, it can be concluded that biocontrol is a promising technique to reduce postharvest losses in lemons.

Key words: lemon, citrus, antagonist, biocontrol, postharvest pathogens.

\section{INTRODUCCIÓN}

Los limones son consumidos en todo el mundo por su sabor fresco, su jugo y su contenido de vitamina C. Desde la cosecha hasta la mesa del consumidor, los limones frescos pasan una cantidad considerable de tiempo en transporte y almacenamiento. Durante estas etapas, los trastornos fisiológicos y las patologías son las principales causas de pérdida de calidad de los frutos. Pretratamientos durante el almacenamiento, como los tipológicos bióticos o abióticos, pueden emplearse para inhibir enfermedades y extender la vida útil (Yun et al., 2013). Las enfermedades se han controlado principalmente mediante la aplicación de fungicidas sintéticos, y otros tratamientos abióticos, los cuales han contribuido a disminuir las pérdidas por daños de fitopatógenos. Sin embargo, los problemas relacionados con el desarrollo de resistencia de patógenos a muchos fungicidas específicos del sitio, los efectos potencialmente nocivos sobre la seguridad humana y la protección del medio ambiente, han estimulado la investigación de métodos alternativos para el control de enfermedades (Zhou et al., 2014a). Es así como se ha establecido la tendencia a utilizar tratamientos bióticos, ya que generalmente son efectivos y no generan residuos sintéticos. El uso de microorganismos antagonistas ha sido efectivo para controlar las enfermedades post-cosecha, con dos enfoques importantes, que consisten en la estimulación y el manejo de los antagonistas presentes sobre la superficie del fruto, y en la introducción artificial de antagonistas contra los patógenos (Hernández-Lauzardo et al., 2007). La superficie del fruto parece ser el mejor lugar para el aislamiento de microorganismos antagonistas, los cuales pueden suprimir el desarrollo de la enfermedad en el fruto.

El presente estudio tiene como objetivo realizar una revisión de las investigaciones recientes relacionadas con el uso de microorganismos beneficiosos o sus derivados para el control de microorganismos patógenos causantes de enfermedades en la post-cosecha del limón como fruto fresco. Aunque también se consideraron estudios realizados en otros cítricos, ya que, muchas de las enfermedades y microorganismos patógenos post-cosecha son similares dentro de este grupo.

\section{MATERIALES Y MÉTODOS}

Se realizó una revisión bibliográfica en las bases de datos Web of Science, ScienceDirect, Scopus, EbscoHost y SpringerLink, de artículos publicados principalmente entre los años 2007 y 2018, con la excepción de algunas publicaciones anteriores que son de importancia en el tema. Se usó Google Académico para contribuir a la obtención de información desde estas bases de datos. Además se usó información desde el sitio web de la Oficina de Estudios y Políticas Agrarias (ODEPA).

\section{RESULTADOS Y DISCUSIÓN}

\section{Importancia del limón en Chile}

Según datos del catastro de ODEPA, en el año 2015 la superficie comercial de la especie limonero fue de aproximadamente $5.516 \mathrm{ha}$, ubicadas principalmente en las regiones Metropolitana, Coquimbo, Valparaíso y O'Higgins. En el año 2016 la superficie fue de aproximadamente 5.313 ha, ubicadas en las regiones anteriormente mencionadas (ODEPA, 2017).

La exportación de limones (Citrus limon L.), frescos o secos, en el año 2015 fue a 18 países, con alrededor de 62.800 toneladas. Dentro de los principales países de destino se encuentran Estados Unidos, Japón, Holanda, Corea del Sur, España, Reino Unido e Italia. En 2016 las exportaciones fueron a más de 20 países, con alrededor de 75.600 toneladas, siendo nuevamente Estados Unidos, Japón y Holanda los principales destinos, seguido por España, Reino Unido, Corea del Sur, Italia y Argentina, entre otros, según el registro de exportaciones anuales de ODEPA (ODEPA, 2017).

La importación de limones ya sea frescos o secos en el año 2015 fue de alrededor de 4.372 toneladas, desde países como Perú y Estados Unidos, principalmente. En el año 2016 se importaron 1.504 toneladas, traídas principalmente desde Estados Unidos y Perú, según el boletín de importaciones anuales de ODEPA (ODEPA, 2017).

El consumo interno de limones frescos en el año 2017 fue alrededor de 95.100 toneladas, considerando la fruta arribada en el mercado mayorista del país, con un precio promedio nominal con IVA de $\$ 1.250$ por kg (ODEPA, 2018). 
Los estándares de calidad de los cítricos para el consumo fresco se basan principalmente en la ausencia de heridas y/o pudriciones (Blasco et al., 2016). Es conocido que las enfermedades post-cosecha causan pérdidas considerables a las frutas y hortalizas. En los países desarrollados se estima que alrededor del $20-25 \%$ de las frutas y hortalizas cosechadas se descomponen por patógenos durante el manejo post-cosecha. En los países en desarrollo, las pérdidas post-cosecha a menudo son más graves debido a instalaciones de almacenamiento y transporte inadecuados (Sharma et al., 2009). Las enfermedades en los limones son causadas principalmente por microorganismos patógenos de post-cosecha en los frutos.

\section{Microorganismos patógenos de importancia en post-cosecha de limones}

Los principales patógenos de los cítricos post cosecha son Penicillium digitatum y Penicillium italicum, agentes causales del moho verde y azul, respectivamente (Zhou et al., 2014a). El moho verde ( $P$. digitatum) es la enfermedad post-cosecha más importante de los limones (Kinay et al., 2007), siendo causante del $90 \%$ de la pérdida de fruta (Macarisin et al., 2007). Ambos patógenos, $P$. digitatum y $P$. italicum, son necrotrofios que requieren heridas para ingresar al fruto a través del flavedo (Ballester et al., 2010). El patógeno P. digitatum tiene un ciclo de enfermedad corto, de tres a cinco días a $25^{\circ} \mathrm{C}$, mientras que el ciclo de $P$. italicum se extiende de siete a diez días a $20^{\circ} \mathrm{C}$ (Askarne et al., 2012). Cada cabeza conidial es capaz de producir hasta 2 billones de conidios y pueden ser propagados por el aire (Sukorini et al., 2013).

Por otra parte, Phyllosticta citricarpa (teleomorfo: Guignardia citricarpa), un hongo ascomiceto, es el agente causal de las manchas negras de limones, que infecta a casi todas las especies económicamente importantes, causando graves pérdidas económicas en la producción de África, Asia, Australia y América del Sur (Wang et al., 2012; Toffano et al., 2017). P. citricarpa infecta las frutas en el campo y puede permanecer en estado inactivo; sin embargo, los frutos asintomáticos pueden desarrollar manchas extensas en la corteza después de la cosecha, muchos meses después de la infección. Aunque la enfermedad no causa decaimiento interno, las frutas se dañan estéticamente para el mercado fresco, y bajo alta intensidad de la enfermedad el rendimiento del cultivo disminuye sustancialmente debido a la caída prematura de la fruta (Guarnaccia et al., 2017). Además, $P$. citricarpa es considerado un patógeno de cuarentena A1 por la Unión Europea, reduciendo considerablemente o haciendo inviable la exportación de cítricos de las zonas productoras donde está presente la mancha negra (Toffano et al., 2017).
Además, en todo el mundo la pudrición causada por Geotrichum citri-aurantii ha sido reportada como una importante enfermedad post-cosecha de cítricos (Regnier et al., 2014). Aunque es menos común que el moho verde y el moho azul, puede causar pérdidas significativas durante los períodos de alta precipitación (Hao et al., 2011). Smilanick et al. (2008) reportaron que ningún fungicida registrado en California, EE.UU., controlaba esta pudrición adecuadamente.

\section{Biocontrol, una solución probable}

La tendencia mundial está cambiando hacia la reducción del uso de fungicidas en los alimentos, y existe gran interés público y científico de buscar alternativas más seguras y ecológicas para reducir la pérdida post-cosecha de las frutas (Mari et al. 2007; Stegmayer et al., 2013).

De varios enfoques biológicos, el uso de microorganismos antagónicos está aumentando en todo el mundo (Marín et al., 2017). Varias enfermedades post-cosecha pueden ahora ser controladas por antagonistas microbianos (Sharma et al., 2009). Los antagonistas microbianos se aplican antes o después de la cosecha, siendo más eficaz la aplicación post-cosecha. El control de las enfermedades en post-cosecha de frutas y hortalizas con microorganismos antagonistas contra patógenos se obtiene bajo dos enfoques básicos: mediante el uso de microorganismos que están presentes naturalmente en la superficie de frutas $\mathrm{y}$ verduras, o bien, introduciendo microorganismos artificialmente. Este último enfoque es más eficaz en el control de enfermedades post-cosecha que otros medios de control biológico (Sharma et al., 2009).

\section{Modo de acción de microorganismos beneficiosos}

El mecanismo o los mecanismos por los cuales los microorganismos beneficiosos ejercen su acción sobre los agentes fitopatógenos todavía no se entienden completamente (Katan, 2017; O'Brien, 2017). Se han sugerido varios modos de acción para explicar la actividad de biocontrol de los antagonistas microbianos. La competencia por nutrientes y espacio entre el patógeno y el antagonista se considera como uno de los principales modos de acción mediante los cuales los agentes microbianos controlan los patógenos que causan la descomposición post-cosecha de fruta (Marín et al., 2017). Además, la resistencia inducida, el parasitismo directo, la adhesión a patógenos y la producción de metabolitos secundarios (antibiosis), son otros modos de acción de los antagonistas microbianos a través de los cuales se suprime la actividad de los patógenos post-cosecha en cítricos (Sharma et al., 2009). Cada microorganismo 
antagonista ejerce inhibición o control de diferente modo, por ejemplo Bacillus subtilis, Pseudomonas syringae, y Trichoderma spp., producen antibióticos, mientras que Debaryomyces hansenii está asociada a la competencia nutricional y la inducción de resistencia del huésped. Según Zhou et al. (2014b), la técnica de inducir la resistencia coincide con la activación de un sistema de defensa bioquímica, que incluye fenilalanina amonio-liasa (PAL), peroxidasa (POD), polifenoloxidasa (PPO), quitinasa (CHI) y $\beta$-1,3-glucanasa, para la resistencia a enfermedades en plantas. PAL es la primera enzima en la vía fenilpropanoide y participa en la biosíntesis de compuestos fenólicos, fitoalexinas y ligninas. POD tiene una función primaria en las etapas finales de la síntesis biológica de lignina. PPO oxida compuestos fenólicos a quininas altamente tóxicas para patógenos invasores. CHI y $\beta$-1,3-glucanasa son enzimas ya bastante caracterizadas que pueden hidrolizar la quitina y $\beta$-1,3-glucano que se encuentran en las paredes celulares de hongos (Luo et al., 2012).

\section{Algunos microorganismos beneficiosos para la post-cosecha de cítricos}

Bacillus spp. han sido reportados como inhibidores del crecimiento de una serie de patógenos vegetales a través de antagonismo, siendo considerados como potenciales agentes de biocontrol, debido a su alta capacidad de producción de esporas, resistencia y capacidad para sobrevivir a la desecación, calor, irradiación ultravioleta (UV) y disolventes orgánicos (Romero et al., 2007). Su principal modo de acción es la competencia por los nutrientes o el espacio, aunque no se puede excluir la interacción directa (Hong et al., 2014). Cepas Ehrenberg Cohn de B. subtilis tienen típicamente la capacidad de sobrevivir en superficies de limones y algunas son antagónicas a patógenos (Abraham et al., 2010). La cepa HF-01 de la bacteria Bacillus amyloliquefaciens puede ser aislada de las superficies de cítricos, caracterizándose por su actividad antifúngica, y por el hecho de ser un antagonista efectivo de los principales patógenos post-cosecha (Hao et al., 2011). Además, es ventajoso y efectivo, ya que al ser un antagonista nativo, la adaptabilidad del antagonista al sustrato natural es particularmente importante (Hao et al., 2011). Del mismo modo, otras bacterias como Pseudomonas spp., presentan actividad de biocontrol contra $P$. digitatum en limones y naranjas ( $\mathrm{Pa}-$ nebianco et al., 2015).

Pichia membranifaciens es una levadura de biocontrol muy conocida, que ha demostrado que controla diversas enfermedades fúngicas post-cosecha de diferentes frutos (Luo et al., 2013). Además de inducir las defensas del huésped, puede suprimir al moho azul y verde de los cítricos, por adhesión a los patógenos y a través de la competencia por los nutrientes, dependiendo del espacio en las heridas de la fruta (Luo et al., 2012; Zhou et al., 2016). Kloeckera apiculata, cepa 34-9, es una levadura antagonista con actividad de control biológico contra las enfermedades post-cosecha de los cítricos (Pu et al., 2014). La cepa CMAA-1112 de la levadura Candida membranifaciens reduce la gravedad de enfermedad a través de la inducción de resistencia en la fruta (Terao et al., 2017). Por otra parte, los hongos Trichoderma spp. presentan actividad de control biológico contra $P$. digitatum (Panebianco et al., 2015).

\section{Inhibición del desarrollo de microorganismos patógenos mediante microorganismos beneficiosos}

Antes de la última década, varios antagonistas microbianos fueron identificados e introducidos artificialmente en productos cosechados. En la Tabla 1 se muestran ejemplos de un control eficaz de la pudrición de los cítricos por mohos, indicando los microorganismos beneficiosos, también llamados antagonistas, que fueron utilizados para el control de enfermedades producidas por microorganismos. Los mecanismos de acción para controlar la descomposición en limones incluyen antibiosis, lisis, competición, micoparasitismo e inducción de la resistencia.

A continuación se describen los resultados más importantes de estudios recientes sobre el uso de microorganismos beneficiosos para inhibir el desarrollo biológico de microorganismos patógenos en limones.

A partir de 205 cepas bacterianas de Bacillus spp., aislados del suelo, se seleccionaron 23 cepas para evaluar su actividad antagónica hacia $P$. digitatum, consiguiendo que los sobrenadantes de cultivo de nueve cepas causaron $>80 \%$ de inhibición del crecimiento de $P$. digitatum cuando se diluyeron en serie a 1:32. Los compuestos volátiles producidos por estas cepas también causaron un $30-70 \%$ de inhibición del crecimiento de hongos. Por otra parte, un extracto de etanol obtenido a partir de un sobrenadante libre de células de $B$. subtilis 155 denominado metabolitos secundarios (SMs) produjo el mejor efecto inhibidor sobre el crecimiento de micelio y la germinación de esporas del hongo, con valores de concentración efectiva media $\mathrm{CE}_{50}$ de 77,26 y $82,10 \mu \mathrm{g} \mathrm{L}^{-1}$, respectivamente. Los compuestos inhibidores fueron separados desde los SMs por cromatografía en capa fina preparativa (Leelasuphakul et al., 2008).

Lai et al. (2012) identificaron la cepa bacteriana SG-6 Paenibacillus polymyxa, aislada como endófita del tejido de raíz de Sophora tonkinensis, obteniendo reducciones significativas del decaimiento post-cosecha de mandarina, originado por $P$. 
Tabla 1. Antagonistas microbianos para el control exitoso de las enfermedades post-cosecha de cítricos.

Table 1. Microbial antagonists for the successful control of postharvest citrus diseases.

\begin{tabular}{|c|c|c|c|}
\hline Antagonista & Enfermedad (patógeno) & Fruta(s) & Referencia(s) \\
\hline Aureobasidium pullulans & Podredumbre (Penicillium spp.) & Naranja & Klein y Kupper, 2018 \\
\hline Bacillus subtilis & Moho verde (Penicillium digitatum) & Limón & Mohammadi et al., 2017 \\
\hline Candida oleophila & $\begin{array}{l}\text { Moho verde (Penicillium digitatum) } \\
\text { Moho azul (Penicillium italicum) } \\
\text { Podredumbre (Geotrichum candidum) }\end{array}$ & $\begin{array}{l}\text { Limón, } \\
\text { mandarina, } \\
\text { naranja }\end{array}$ & $\begin{array}{l}\text { Lahlali et al., 2004; } \\
\text { Ballet et al., } 2016\end{array}$ \\
\hline Debaryomyces hansenii & Moho azul (Penicillium italicum) & Limón & $\begin{array}{l}\text { Hernández-Montiel et } \\
\text { al., 2010; } 2012\end{array}$ \\
\hline Pantoea agglomerans & $\begin{array}{l}\text { Moho verde (Penicillium digitatum) } \\
\text { Moho azul (Penicillium italicum) }\end{array}$ & $\begin{array}{l}\text { Mandarina, } \\
\text { naranja }\end{array}$ & $\begin{array}{l}\text { Teixidó et al., 2001; } \\
\text { Torres et al., 2007; } 2014\end{array}$ \\
\hline Pichia anomala & $\begin{array}{l}\text { Moho verde (Penicillium digitatum) } \\
\text { Moho azul (Penicillium italicum) } \\
\text { Podredumbre (Geotrichum candidum) }\end{array}$ & $\begin{array}{l}\text { Mandarina, } \\
\text { naranja }\end{array}$ & $\begin{array}{l}\text { Lahlali et al., 2004; } \\
\text { Taqarort et al., } 2008\end{array}$ \\
\hline Pichia guilliermondii & $\begin{array}{l}\text { Moho verde (Penicillium digitatum) } \\
\text { Moho azul (Penicillium italicum) }\end{array}$ & Naranja & $\begin{array}{l}\text { Sangwanich et al., 2013; } \\
\text { Lahlali et al., } 2014\end{array}$ \\
\hline Pseudomonas cepacia & Moho verde (Penicillium digitatum) & Naranja & Huang et al., 1993 \\
\hline Pseudomonas syringae & Moho verde (Penicillium digitatum) & $\begin{array}{l}\text { Limón, } \\
\text { naranja }\end{array}$ & Panebianco et al., 2015;2016 \\
\hline Trichoderma atroviride & Moho verde (Penicillium digitatum) & $\begin{array}{l}\text { Limón, } \\
\text { naranja }\end{array}$ & Panebianco et al., 2015 \\
\hline
\end{tabular}

digitatum y P. italicum. Esta cepa era altamente efectiva en la reducción de la descomposición de los cítricos causada por $P$. digitatum. El control del moho verde mejoró cuando aumentó el período entre el tratamiento con la cepa SG-6 y la inoculación con el patógeno. La concentración del antagonista también tuvo un efecto significativo sobre la eficacia del biocontrol. A la concentración de 1 x $10^{9}$ células $\mathrm{mL}^{-1}$ de la cepa SG-6, la incidencia de la enfermedad y el diámetro de la lesión disminuyeron hasta $18,3 \%$ y $5,5 \mathrm{~mm}$, respectivamente. Además, no afectó los parámetros de calidad, como pérdida de peso, firmeza, sólidos solubles totales, ácido ascórbico y acidez titulable.

Abraham et al. (2010) obtuvieron 92 aislamientos de Bacillus y 60 levaduras de la superficie de la fruta de papaya y de diversas variedades de cítricos de varios huertos en Sudáfrica, y las analizaron para antagonismo a $P$. digitatum. Se obtuvieron dos aislados de levadura (B13 y Uva), que cuando se aplicaron 48 horas antes de la inoculación con $P$. digitatum, evitaban la descomposición de naranjas y limones. Ambas levaduras redujeron los diámetros de lesión de $P$. digitatum en el limón, independientemente de las concentraciones. El aislamiento B13 demostró una mayor eficacia de biocontrol y un desarrollo completamen- te controlado de $P$. digitatum a $1 \times 10^{8}$ células $\mathrm{mL}^{-1}$. Indican además que si las esporas de Penicillium tienen acceso a los nutrientes de la herida durante 3 horas antes de que se apliquen las levaduras, entonces la estimulación de las esporas de Penicillium para germinar ya habrá ocurrido. Por lo tanto, los aislados de levaduras no serían eficaces en un papel curativo.

Una potencial levadura de biocontrol, Rhodosporidium paludigenum, fue investigada por $\mathrm{Lu}$ et al. (2013a) por su inducción de resistencia a la enfermedad contra $P$. digitatum en los cítricos. Los resultados de este estudio muestran que la capacidad de R. paludigenum para estimular la resistencia a enfermedades depende del momento de la inoculación de la levadura. El porcentaje de infección y la gravedad de la enfermedad disminuyeron significativamente con el aumento del tiempo de incubación. Se consideró $10^{8}$ células $\mathrm{mL}^{-1}$ como la concentración óptima de incubación de levadura para provocar resistencia a $P$. digitatum. Esto indica que la resistencia inducida por $R$. paludigenum sólo puede proteger la fruta de una infección posterior después del tratamiento, induciendo mayores niveles de resistencia a la enfermedad contra $P$. digitatum y mejorando significativamente las actividades de las enzimas re- 
lacionadas con la defensa. Luego, Lu et al. (2013b) utilizaron $R$. paludigenum para evaluar la eficacia de las aplicaciones pre-cosecha contra las enfermedades post-cosecha en mandarina.

Luo et al. (2012) examinaron la capacidad de la levadura $P$. membranifaciens para controlar $P$. italicum y $P$. digitatum durante el almacenamiento, notando una menor incidencia de enfermedades en frutos cítricos sumergidos en $1 \times 10^{8} \mathrm{CFU} \mathrm{mL}^{-1}$ de $P$. membranifaciens. El contenido de compuestos fenólicos, flavonoides, POD, PPO, PAL, CHI y $\beta$-1,3-glucanasa en cáscaras de cítricos aumentó en frutos sumergidos o inoculados con $P$. membranifaciens, lo cual coincidió con el inicio de la resistencia inducida. La competencia por nutrientes y el espacio en heridas, y las defensas inducidas del huésped parecen contribuir al mecanismo de control de P. membranifaciens (Zhou et al., 2016). Luo et al. (2013) evaluaron los efectos de P. membranifaciens sobre el metabolismo de las especies reactivas de oxígeno (ROS) y el control de enfermedades en cítricos cosechados (Citrus sinensis $\mathrm{L}$. Osbeck), obteniendo que el diámetro de la lesión causada por $P$. italicum y $P$. digitatum se redujo notablemente cuando la fruta era inoculada en un punto o sumergida en una suspensión de $P$. membranifaciens a $1 \times 10^{8} \mathrm{CFU} \mathrm{mL}^{-1}$. En este estudio la aplicación de $P$. membranifaciens en los cítricos mejoró la actividad de superóxido dismutasa, ascorbato peroxidasa y glutatión reductasa, así como los niveles de peróxido de hidrógeno, el anión superóxido y el glutatión, pero inhibió la disminución del contenido de ácido ascórbico. Además, la actividad de la catalasa se redujo mediante el mismo tratamiento. Estos resultados indicaron que el tratamiento con la levadura indujo la síntesis de enzimas oxido-reductasas que podrían tener efectos antagonistas contra la infección de moho verde y azul.

Liu et al. (2017) exploraron la diversidad de levaduras de naranjas en plantaciones del distrito Beibei, Chongqing, y su potencial para el control biológico de $P$. digitatum en las naranjas. Las levaduras se aislaron de la superficie de frutas, hojas y tierra, obteniendo 71 cepas de levadura que pertenecían a ocho géneros: Metschnikowia, Pichia, Candida, Pseudozyma, Kazachstania, Issatchenkia, Hanseniaspora, y Barnettozyma. Todos los aislados de levadura se evaluaron para la inhibición de moho verde en un medio de agar de dextrosa de papa (PDA), y 45 aislamientos redujeron el crecimiento del micelio fúngico. Se analizaron doce aislamientos para determinar la actividad antagonista contra $P$. digitatum, y la actividad de control biológico de los diferentes aislamientos en la reducción de la incidencia de la enfermedad varió entre 26 y $93 \%$. En particular revelaron que Metschnikowia sp. FL02 inhibió completamente la aparición de la putrefacción del moho verde el séptimo día después de la inoculación.

$\mathrm{Pu}$ et al. (2014) utilizaron la levadura K. apiculata cepa 34-9 para el control biológico; en estudios anteriores se encontró que $K$. apiculata producía alcohol aromático feniletanol, y en el presente estudio encontraron que $K$. apiculata se adhería y formaba un biofilm en la superficie de los cítricos, lo que creó una barrera mecánica interpuesta entre la superficie de la herida y el patógeno. Como molécula de detección de quórum (QS), el feniletanol puede promover la formación de filamentos por K. apiculata en medio de agar de dextrosa de papa, mientras que en los cítricos, el antagonista permanece como levadura después de ser tratado con la misma concentración de feniletanol. Este sólo indujo a K. apiculata para adherirse y formar un biofilm. Se identificaron nueve genes posiblemente implicados en el desencadenamiento de la adhesión de levadura; seis de estos genes se indujeron significativamente después del tratamiento de estrés con feniletanol. Este estudio propone la levadura antagonista K. apiculata como modelo biológico para estudiar las interacciones antagonista-patógeno y el control del moho azul en cítricos. Asimismo, los compuestos orgánicos volátiles (COVs) de origen microbiano son relevantes en las interacciones biológicas y se consideran agentes ambientalmente más seguros para controlar las enfermedades post-cosecha de los frutos.

Toffano et al. (2017) evaluaron la efectividad de los COVs producidos por Saccharomyces cerevisiae para controlar el hongo filamentoso Phyllosticta citricarpa; e informaron que $P$. citricarpa expuesto a la mezcla sintética de estos COVs, afecta negativamente al fitopatógeno. Individualmente, el 3-metil-1-butanol y el 2-metil-1-butanol fueron los COVs más efectivos que inhibieron completamente el crecimiento de micelio y la germinación y formación de appressorium por conidios.

Diversas investigaciones están orientadas a descubrir nuevos microorganismos en zonas extremas como fuente de biodiversidad para el aislamiento y selección de microorganismos útiles. Entre estas investigaciones, destaca la realizada por Oro et al. (2016) quienes aislaron, desde diferentes sustratos del desierto de Namibia, aproximadamente 44 levaduras, las cuales mostraron actividad antifúngica contra diferentes hongos fitopatógenos y entre ellos $P$. digitatum. Entre estas cepas destacaron Aureobasidium pullulans (cepas F32 y F60), Cryptococcus albidus (S14) y S. cerevisiae (RB24) las cuales inhibieron considerablemente a $P$. digitatum en limones y naranjas.

Recientemente Perez et al. (2016; 2017), reportaron el uso de levaduras aisladas desde hojas y 
frutos de limón con el objetivo de controlar in vitro e in vivo a P. digitatum y $P$. italicum. Del total de 437 levaduras aisladas, alrededor del 8\% mostró actividad mortífera, destacándose las cepas Clavispora lusitaniae-146 y Pichia fermentans-27, las cuales mostraron una alta eficiencia para proteger los frutos de limón de los patógenos, a las diferentes temperaturas evaluadas. Además, fueron más eficientes comparadas con el control biológico comercial basado en Candida oleophila. Ambas cepas fueron tolerantes a fungicidas comerciales, por lo cual el uso combinado de agentes químicos y de estas levaduras podrían ser empleados en post-cosecha de limón.

Suwannarach et al. (2013) aislaron 46 endófitos fúngicos desde Lagerstroemia loudoni y encontraron un solo hongo que producía compuestos volátiles antifúngicos, identificado como Nodulisporium spp. CMU-UPE34, que produjo 31 compuestos volátiles, principalmente alcoholes, ácidos, ésteres y monoterpenos. El compuesto volátil más abundante fue el eucaliptol. Por otra parte, Nodulisporium spp. CMU-UPE34 controló el deterioro por moho verde en Citrus limon causado por $P$. digitatum, y la descomposición por moho azul de Citrus aurantifolia y Citrus reticulata causada por $P$. expansum.

Panebianco et al. (2015) explicaron que las mezclas de células de Pseudomonas y filtrados de cultivo de Trichoderma atroviride P1 incrementaron los efectos inhibidores. Por otra parte, la mezcla de los filtrados bacterianos y fúngicos nunca indujo una eficacia mejorada, indicando así que se necesita la presencia de células bacterianas vivas para un efecto sinérgico. Por lo tanto, la incidencia y gravedad de la enfermedad en limón cv. Femminello se redujo consistentemente cuando las cepas de Pseudomonas y Trichoderma se aplicaron 72 horas antes de la inoculación del patógeno a $P$. digitatum. Las cepas de $P$. syringae fueron las más eficaces; además, la combinación de cepas resultó una actividad de biocontrol superior a la de cada uno de los microorganismos antagonistas aplicados de manera separada. Los tratamientos que comprendían seis cepas de $P$. syringae en combinación con las cepas T22, P1 y T34 de Trichoderma fueron los más efectivos, con un $80-100 \%$ de control de moho verde. Los autores sugieren que la combinación de Pseudomonas spp. y Trichoderma spp., podría ser considerada como una medida promisoria para el control de la descomposición del moho verde de limones.

\section{Combinación de microorganismos beneficiosos y otras técnicas para el control de fitopatógenos}

La eficacia del biocontrol y la estabilidad de las levaduras antagonistas por sí solas no son equivalentes a las de los fungicidas sintéticos (Droby et al., 2009). Por lo tanto, el rendimiento de los microorganismos de biocontrol debe ser mejorado usando una combinación de microorganismos antagonistas con otras técnicas alternativas.

El tratamiento con agua caliente (HWT) mejoró la eficacia de $P$. membranifaciens en el control de las enfermedades post-cosecha, induciendo la acumulación de PAL, POD, PPO, CHI, y $\beta$-1,3-glucanasa, así como el contenido total de compuestos fenólicos (Yun et al., 2013; Zhou et al. 2014a). La integración de antagonistas con tratamientos de agua caliente podría mejorar la bioeficacia de los antagonistas microbianos tales como B. subtilis, P. syringae, Cryptococcus laurentii (Zhang et al., 2007), Pantoea agglomerans (Torres et al., 2007) y Pichia guilliermondii (Liu et al., 2010). Zhou et al. (2014b) demostraron que P. membranifaciens combinado con ácido salicílico (SA) proporcionó una rápida y fuerte inducción de las reacciones relacionadas con la defensa (generando la activación del sistema de defensa bioquímica, que incluye PAL, POD, PPO, CHI y $\beta$-1,3-glucanasa), lo que puede correlacionarse con la menor incidencia de infección y el diámetro de la lesión. La combinación de $P$. membranifaciens con SA $\left(10 \mu \mathrm{g} \mathrm{mL}^{-1}\right)$ demostró ser una estrategia más eficaz para controlar los mohos azules y verdes post-cosecha que la aplicación por separado de $P$. membranifaciens o SA. P. membranifaciens más SA indujeron notablemente la acumulación de PAL, POD, PPO, CHI y $\beta$-1,3-glucanasa, así como el contenido total de compuestos fenólicos.

Hong et al. (2014) evaluaron los efectos sobre microorganismos patógenos de un aislado antagonista Bacillus amyloliquefaciens HF-01, de bicarbonato de sodio (SBC) y de un tratamiento de agua caliente (HW), individualmente y en combinación. Los resultados revelaron que la actividad biológica de $B$. amyloliquefaciens HF-01 contra $P$. italicum, $P$. digitatum y G. citri-aurantii en fruta de mandarina fue mejorada en gran medida por la combinación de una dosis baja de $\mathrm{SBC}$ a $6^{\circ} \mathrm{C}$ o $25^{\circ} \mathrm{C}$. Además, mediante la combinación del tratamiento con agua caliente y $B$. amyloliquefaciens HF-01, se redujo notablemente el diámetro de la lesión y la incidencia de la enfermedad de los mohos verdes y azules. Por otra parte, se ha encontrado que el SBC ha sido muy exitoso cuando se usa con el antagonista microbiano $P$. agglomerans (Torres et al., 2007; Usall et al., 2008).

Torres et al. (2007) determinaron el potencial de utilizar un producto basado en $P$. agglomerans CPA-2, solo o en combinación con soluciones de bicarbonato de sodio caliente para controlar las principales enfermedades post-cosecha que afectan a los cultivos de cítricos en la región mediterránea. Aplicaciones de CPA-2 liofilizada y una solución de $\mathrm{SBC}$ al $3 \%$ a $50^{\circ} \mathrm{C}$ durante 40 
segundos proporcionaron un mejor control de la descomposición en mandarinas no dañadas y naranjas artificialmente inoculadas tanto con $P$. digitatum como con $P$. italicum.

Guo et al. (2014) evaluaron la actividad preventiva del metil jasmonato (MeJA) en combinación con levadura antagonista C. laurentii. Se obtuvo que la aplicación preventiva de C. laurentii en $1 \times 10^{8}$ células $\mathrm{mL}^{-1}$ en combinación con 100 $\mu \mathrm{mol} \mathrm{L} \mathrm{L}^{-1}$ MeJA logró la reducción de la incidencia del moho verde comparado con el control, en mandarinas heridas inoculadas con $P$. digitatum, es decir, la combinación de MeJA con C. laurentii es eficaz para reducir la incidencia de moho verde. El mecanismo sugiere que MeJA y C. laurentii combinados inducen la resistencia natural y estimulan el crecimiento de levadura antagonista en la superficie del fruto.

Una combinación de un extracto vegetal prometedor de Eugenia caryophyllata y levadura Candida utilis TISTR 5001 fue investigada por Sukorini et al. (2013) para el control de la enfermedad de $P$. digitatum en cítricos. Los resultados indicaron que la combinación de extracto crudo de E. caryophyllata y C. utilis TISTR 5001 redujo significativamente el desarrollo natural de la incidencia de pudrición del moho verde en un $90 \%$, y no tuvo ningún efecto dañino en la calidad del fruto; por otra parte, se encontró que el compuesto activo de E. caryophyllata era eugenol.

Hao et al. (2011) evaluaron una cepa de B. amyloliquefaciens HF-01 en combinación con saponina de té (TS). El tratamiento que comprendía HF-01 combinado con $50 \mu \mathrm{g} \mathrm{mL}^{-1}$ TS fue tan eficaz como el tratamiento con fungicida, dio más del $90 \%$ de control del moho verde y azul, y la pudrición amarga, sin afectar a ninguno de los otros parámetros de calidad del fruto.

Terao et al. (2017) usaron la combinación de tratamientos físicos (cepillado con agua caliente (HWB) y la irradiación ultravioleta C (UVC)) con C. membranifaciens CMAA-1112 para controlar el moho verde en fruta crítica, donde C. membranifaciens presentó un efecto aditivo aumentando la eficacia en el control de la enfermedad, y extendió la vida útil de la fruta.

Klein y Kupper (2018) estudiaron la eficacia del biocontrol de la cepa ACBL-77 de A. pullulans contra Geotrichum citri-aurantii, incorporando nutrientes para evaluar la competencia por nutrientes entre microorganismos. Indicaron que las fuentes de micronutrientes (ácido bórico, cloruro de cobalto y molibdato de amonio) favorecían la acción antagonista de A. pullulans. El sulfato de amonio al $1 \%$ y sacarosa $0,5 \%$ favorecieron la levadura durante la competición entre los microorganismos. La adición de sulfato de amonio (1\%) en el cultivo de levaduras estimuló la producción de biofilm y aumentó la actividad antagonista contra la enfermedad, así como permitió una mejor supervivencia de la levadura. Se encontró que la levadura era capaz de formar biofilms, deformando las hifas patógenas.

Usall et al. (2008) presentan la actividad preventiva y curativa de los tratamientos de inmersión en soluciones acuosas a $40^{\circ} \mathrm{C}$, de carbonato de sodio (SC) al 3\% o SBC; solo o en combinación con $P$. agglomerans CPA-2 (BA) para el control del moho verde causado por $P$. digitatum en limones. Encontraron que las células frescas de BA proliferaron dentro de las heridas de corteza y su supervivencia no fue afectada negativamente por la presencia de residuos de SC o SBC. El efecto protector de SBC fue significativamente inferior al efecto de $P$. agglomerans CPA-2 y al efecto de SBC combinado con BA, señalando que la integración de tratamientos es un enfoque prometedor para reemplazar el uso de fungicidas convencionales.

Long et al. (2007) evaluaron la eficacia de la levadura K. apiculata cepa 34-9 para controlar la incidencia natural de la descomposición post-cosecha de cítricos en combinación con una baja concentración de fungicida, consiguiendo inhibir eficazmente el desarrollo de la desintegración en la fruta. En pruebas de empaque, la combinación de la levadura con $40 \mathrm{mg} \mathrm{L}^{-1}$ de Carbendazim dio como resultado una reducción en la incidencia de heridas, causada por los mohos verde y azul, igual a un tratamiento fungicida convencional, proponiendo que la eficacia de K. apiculata cepa 34-9 en condiciones de envasado a una concentración celular de la levadura antagonista $3 \times 10^{8}$ células $\mathrm{mL}^{-1}$ y señalando que la cepa no alteró ningún parámetro de calidad de la fruta cítrica.

\section{CONCLUSIONES}

En post-cosecha de limones, los microorganismos Bacillus (B. amyloliquefaciens; $B$. subtilis) Pseudomonas (P. syringae), P. agglomerans, $P$. polymyxa, C. laurentii, C. utilis, C. membranifaciens, D. hansenii, K. apiculata, Metschnikowia spp., P. membranifaciens, P. guilliermondii. S. cerevisiae, $R$. paludigenum, A. pullulans, Nodulisporium spp., y Trichoderma spp., han presentado resultados positivos en la disminución del desarrollo biológico de microorganismos patógenos como $P$. digitatum, P. italicum, P. citricarpa, y G. citri-aurantii, sin provocar daños a la calidad del fruto con su utilización. En la revisión bibliográfica estos microorganismos beneficiosos son considerados prometedores para el control de patógenos a escala comercial. Sin embargo, ninguno de ellos, por sí solo o en asociación con otros agentes de naturaleza fisicoquímica, se ha impuesto aún según el estado del arte. Finalmente, se requiere avanzar 
en estudios comparativos, para posteriormente realizar ensayos de aplicación a nivel piloto, que permitan concluir respecto de la factibilidad técnica y económica de los métodos de biocontrol desarrollados.

\section{LITERATURA CITADA}

Abraham, A.O., M.D. Laing, and J.P. Bower. 2010. Isolation and in vivo screening of yeast and Bacillus antagonists for the control of Penicillium digitatum of citrus fruit. Biol. Control 53:32-38. doi:10.1016/j.biocontrol.2009.12.009

Askarne, L., I. Talibi, H. Boubaker, E.H. Boudyach, F. Msanda, B. Saadi, et al. 2012. In vitro and in vivo antifungal activity of several Moroccan plants against Penicillium italicum, the causal agent of citrus blue mold. Crop Prot. 40:53-58. doi:10.1016/j.cropro.2012.04.023

Ballester, A.R., A. Izquierdo, M.T. Lafuente, and L. González-Candelas. 2010. Biochemical and molecular characterization of induced resistance against Penicillium digitatum in citrus fruit. Postharvest Biol. Tec. 56:31-38. doi:10.1016/j.postharvbio.2009.10.002

Ballet, N., J.L. Souche, and P. Vandekerckove. 2016. Efficacy of Candida oleophila, strain O, in preventing postharvest diseases of fruits. Acta Hortic. 1144:105-111. doi:10.17660/ActaHortic.2016.1144.15

Blasco, J., S. Cubero, and E. Moltó. 2016. Quality evaluation of citrus fruits. p. 305-325. In D.W. Sun (ed.) Computer vision technology for food quality evaluation. 2nd ed. Academic Press, San Diego, USA. doi:10.1016/B978-012-802232-0.00012-8

Droby, S., M. Wisniewski, D. Macarisin, and C. Wilson. 2009. Twenty years of postharvest biocontrol research: Is it time for a new paradigm? Postharvest Biol. Tec. 52:137-145. doi:10.1016/j.postharvbio.2008.11.009

Guarnaccia, V., J.Z. Groenewald, H. Li, C. Glienke, E. Carstens, V. Hattingh, and P.W. Crous. 2017. First report of Phyllosticta citricarpa and description of two new species, $P$. paracapitalensis and P. paracitricarpa, from citrus in Europe. Stud. Mycol. 87:161-185. doi:10.1016/j. simyco.2017.05.003

Guo, J., W. Fang, H. Lu, R. Zhu, L. Lu, X. Zheng, et al. 2014. Inhibition of green mold disease in mandarins by preventive applications of methyl jasmonate and antagonistic yeast Cryptococcus laurentii. Postharvest Biol. Tec. 88:72-78. doi:10.1016/j.postharvbio.2013.09.008
Hao, W., H. Li, M. Hu, L. Yang, and M. Rizwan-ulHaq. 2011. Integrated control of citrus green and blue mold and sour rot by Bacillus amyloliquefaciens in combination with tea saponin. Postharvest Biol. Tec. 59:316-323. doi:10.1016/j. postharvbio.2010.10.002

Hernández-Lauzardo, A.N., S. Bautista-Baños, M.G. Velásquez-del Valle, and A. Hernández-Rodríguez. 2007. Uso de microorganismos antagonistas en el control de enfermedades postcosecha en frutos. Rev. Mex. Fitopatol. 25(1):66-74.

Hernández-Montiel, L.G., J.L. Ochoa, E. Troyo-Diéguez, and C.P. Larralde-Corona. 2010. Biocontrol of postharvest blue mold (Penicillium italicum Wehmer) on Mexican lime by marine and citrus Debaryomyces hansenii isolates. Postharvest Biol. Tec. 56:181-187. doi:10.1016/j. postharvbio.2009.12.010

Hernández-Montiel, L.G., R.J. Holguín-Peña, C.P. Larralde-Corona, R. Zulueta-Rodríguez, E. Rueda-Puente, and M. Moreno-Legorreta. 2012. Effect of inoculum size of yeast Debaryomyces hansenii to control Penicillium italicum on Mexican lime (Citrus aurantiifolia) during storage. CYTA J. Food 10:235-242. doi:10.108 0/19476337.2011.633350

Hong, P., W. Hao, J. Luo, S. Chen, M. Hu, and G. Zhong. 2014. Combination of hot water, Bacillus amyloliquefaciens HF-01 and sodium bicarbonate treatments to control postharvest decay of mandarin fruit. Postharvest Biol. Tec. 88:96-102. doi:10.1016/j.postharvbio.2013.10.004

Huang, Y., B.J. Deverall, and S.C. Morris. 1993. Effect of Pseudomonas cepacia on postharvest biocontrol of infection by Penicillium digitatum and on wound response of citrus fruit. Aust. Plant Pathol. 22:84-93. doi:10.1071/ APP9930084

Kinay, P., M.F. Mansour, F.M. Gabler, D.A. Margosan, and J.L. Smilanick. 2007. Characterization of fungicide-resistant isolates of Penicillium digitatum collected in California. Crop Prot. 26:647-656. doi:10.1016/j.cropro.2006.06.002

Katan, J. 2017. Diseases caused by soilborne pathogens: Biology, management and challenges. J. Plant Pathol. 99(2):305-315.

Klein, M.N., and K.C. Kupper. 2018. Biofilm production by Aureobasidium pullulans improves biocontrol against sour rot in citrus. Food Microbiol. 69:1-10. doi:10.1016/j.fm.2017.07.008

Lahlali, R., M.N. Serrhini, and M.H. Jijakli, 2004. Efficacy assessment of Candida oleophila (strain $\mathrm{O}$ ) and Pichia anomala (strain K) against major postharvest diseases of citrus fruits in Morocco. Comm. Agric. Appl. Biol. Sci. 69(4):601609. 
Lahlali, R., Y. Hamadi, R. Drider, C. Misson, M. El Guilli, and M.H. Jijakli, 2014. Control of citrus blue mold by the antagonist yeast Pichia guilliermondii Z1: Compatibility with commercial fruit waxes and putative mechanisms of action. Food Control 45:1-7. doi:10.1016/j. foodcont.2014.04.014

Lai, K., S. Chen, M. Hu, Q. Hu, P. Geng, Q. Weng, et al. 2012. Control of postharvest green mold of citrus fruit by application of endophytic Paenibacillus polymyxa strain SG-6. Postharvest Biol. Tec. 69:40-48. doi:10.1016/j.postharvbio.2012.03.001

Leelasuphakul, W., P. Hemmanee, and S. Chuenchitt. 2008. Growth inhibitory properties of Bacillus subtilis strains and their metabolites against the green mold pathogen (Penicillium digitatum Sacc.) of citrus fruit. Postharvest Biol. Tec. 48:113-121. doi:10.1016/j.postharvbio.2007.09.024

Liu, F., K. Tu, X. Shao, Y. Zhao, S. Tu, J. Su et al. 2010. Effect of hot air treatment in combination with Pichia guilliermondii on postharvest anthracnose rot of loquat fruit. Postharvest Biol. Tec. 58:65-71. doi:10.1016/j.postharvbio.2010.05.009

Liu, Y., W. Wang, Y. Zhou, S. Yao, L. Deng, and K. Zeng. 2017. Isolation, identification and in vitro screening of Chongqing orangery yeasts for the biocontrol of Penicillium digitatum on citrus fruit. Biol. Control 110:18-24. doi:10.1016/j.biocontrol.2017.04.002

Long, C.A., B.X. Deng, and X.X. Deng. 2007. Commercial testing of Kloeckera apiculata, isolate 34-9, for biological control of postharvest diseases of citrus fruit. Ann. Microbiol. 57:203207. doi:10.1007/BF03175208

Lu, L., H. Lu, C. Wu, W. Fang, C. Yu, C. Ye, et al. 2013a. Rhodosporidium paludigenum induces resistance and defense-related responses against Penicillium digitatum in citrus fruit. Postharvest Biol. Tec. 85:196-202. doi:10.1016/j.postharvbio.2013.06.014

Lu, L., C. Ye, S. Guo, K. Sheng, L. Shao, T. Zhou et al. 2013b. Preharvest application of antagonistic yeast Rhodosporidium paludigenum induced resistance against postharvest diseases in mandarin orange. Biol. Control 67:130-136. doi:10.1016/j.biocontrol.2013.07.016

Luo, Y., K. Zeng, and J. Ming. 2012. Control of blue and green mold decay of citrus fruit by Pichia membranefaciens and induction of defense responses. Sci. Hortic. 135:120-127. doi:10.1016/j.scienta.2011.11.031
Luo, Y., Y. Zhou, and K. Zeng. 2013. Effect of Pichia membranaefaciens on ROS metabolism and postharvest disease control in citrus fruit. Crop Prot. 53:96-102. doi:10.1016/j.cropro.2013.06.015

Macarisin, D., L. Cohen, A. Eick, G. Rafael, E. Belausov, M. Wisniewski et al. 2007. Penicillium digitatum suppresses production of hydrogen peroxide in host tissue during infection of citrus fruit. Phytopathology 97:1491-1500. doi:10.1094/PHYTO-97-11-1491

Mari, M., F. Neri, and P. Bertolini. 2007. Novel approaches to prevent and control postharvest diseases of fruits. Stewart Postharvest Rev. 6(4):1-7. doi:10.2212/spr.2007.6.4

Marín, A., L. Atarés, and A. Chiralt. 2017. Improving function of biocontrol agents incorporated in antifungal fruit coatings: a review. Biocontrol Sci. Techn. 27(10):1220-1241. doi:10.1 080/09583157.2017.1390068

Mohammadi, P., E. Tozlu, R. Kotan, and M. Şenol Kotan. 2017. Potential of some bacteria for biological control of postharvest citrus green mould caused by Penicillium digitatum. Plant Prot. Sci. 53:134-143. doi:10.17221/55/2016PPS

O'Brien, P.A. 2017. Biological control of plant diseases. Australas. Plant Pathol. 46:293-304. doi: 10.1007/s13313-017-0481-4

ODEPA. 2017. Boletín fruta fresca. Mayo de 2017. Oficina de Estudios y Políticas Agrarias (ODEPA), Santiago, Chile. Disponible en http://www.odepa.cl/ boletin/boletin-fruta-fresca-mayo-de-2017/ (Consulta 12 junio 2017).

ODEPA. 2018. Oficina de Estudios y Políticas Agrarias (ODEPA), Santiago, Chile. Disponible en http://www.odepa.gob.cl. (Consulta 9 marzo 2018).

Oro, L., M. Ciani, F. Comitini. 2016. Yeasts from xerophilic environments reveal antimicrobial action against fruit pathogenic molds. J. Food Safety. 36:100-108. doi: 10.1111/ jfs.12217 10.1016

Panebianco, S., A. Vitale, G. Polizzi, F. Scala, and G. Cirvilleri. 2015. Enhanced control of postharvest citrus fruit decay by means of the combined use of compatible biocontrol agents. Biol. Control 84:19-27. doi:10.1016/j. biocontrol.2015.02.001

Panebianco, S., A. Vitale, G. Polizzi, C. Restuccia, and G. Cirvilleri. 2016. Control of Penicillium digitatum on "Tarocco" orange by combined application of pseudomonas syringae and resistance inducers. Acta Hortic. 1144:431-438. doi:10.17660/ActaHortic.2016.1144.64 
Perez, M.F., L. Contreras, N.M. Garnica, M.V. Fernández-Zenoff, M.E. Farías, M. Sepulveda et al. 2016. Native killer yeasts as biocontrol agents of postharvest fungal diseases in lemons. PLOS ONE 11(10):e0165590. Doi:10.1371/journal.pone.0165590

Perez, M.F., J. Perez Ibarreche, A.S. Isas, M. Sepulveda, J. Ramallo, J.R. Dib. 2017. Antagonistic yeasts for the biological control of Penicillium digitatum on lemons stored under export conditions. Biol. Control 115:135-140. doi. org/10.1016/j.biocontrol.2017.10.006

$\mathrm{Pu}, \mathrm{L}$., F. Jingfan, C. Kai, L. Chao-an, and C. Yunjiang. 2014. Phenylethanol promotes adhesion and biofilm formation of the antagonistic yeast Kloeckera apiculata for the control of blue mold on citrus. FEMS Yeast Res. 14:536546. doi:10.1111/1567-1364.12139

Regnier, T., S. Combrinck, W. Veldman and W. Du Plooy. 2014. Application of essential oils as multi-target fungicide for the control of Geotrichum citri-aurantii and other postharvest pathogens of citrus. Ind. Crops Prod. 61:151-159. doi:10.1016/j.indcrop.2014.05.052

Romero, D., A. de Vicente, R.H. Rakotoaly, S.E. Dufour, J.W. Veening, E. Arrebola et al. 2007. The iturin and fengycin families of lipopeptides are key factors in antagonism of Bacillus subtilis toward Podosphaera fusca. Mol. Plant Microbe Int. 20:430-440. doi:10.1094/MPMI20-4-0430

Sangwanich, S., S. Sangchote, and W. Leelasuphakul. 2013. Biocontrol of citrus green mold and postharvest quality parameters. Int. Food Res. J. 20:3381-3386.

Sharma, R.R., D. Singh, and R. Singh. 2009. Biological control of postharvest diseases of fruits and vegetables by microbial antagonists: A review. Biol. Control 50:205-221. doi:10.1016/j.biocontrol.2009.05.001

Smilanick, J.L., M.F. Mansour, F.M. Gabler, and D. Sorenson. 2008. Control of citrus postharvest green mold and sour rot by potassium sorbate combined with heat and fungicides. Postharvest Biol. Tec. 47:226-238. doi:10.1016/j.postharvbio.2007.06.020

Stegmayer, G., D.H. Milone, S. Garran, and L. Burdyn. 2013. Automatic recognition of quarantine citrus diseases. Expert Syst. Appl. 40:3512-3517. doi:10.1016/j.eswa.2012.12.059

Sukorini, H., S. Sangchote, and N. Khewkhom. 2013. Control of postharvest green mold of citrus fruit with yeasts, medicinal plants, and their combination. Postharvest Biol. Tec. 79:24-31. doi:10.1016/j.postharvbio.2013.01.001
Suwannarach, N., J. Kumla, B. Bussaban, W. Nuangmek, K. Matsui, and S. Lumyong. 2013. Biofumigation with the endophytic fungus Nodulisporium spp. CMU-UPE34 to control postharvest decay of citrus fruit. Crop Prot. 45:63-70. doi:10.1016/j.cropro.2012.11.015

Taqarort, N., A. Echairi, R. Chaussod, R. Nouaim, H. Boubaker, A.A. Benaourmar, et al. 2008. Screening and identification of epiphytic yeasts with potential for biological control of green mold of citrus fruits. World J. Microb. Biot. 24:3031-3038. doi:10.1007/s11274-0089849-5

Terao, D., K. de Lima Nechet, M.S. Ponte, A. de Holanda Nunes Maia, V. Delgado de Almeida Anjos, et al. 2017. Physical postharvest treatments combined with antagonistic yeast on the control of orange green mold. Sci. Hortic. 224:317-323. doi:10.1016/j.scienta.2017.06.038

Teixidó, N., J. Usall, L. Palou, A. Asensio, C. Nunes, and I. Viñas. 2001. Improving control of green and blue molds of oranges by combining Pantoea aglomerans (CPA-2) and sodium bicarbonate. Eur. J. Plant Pathol. 107(7):685-694.

Toffano, L., M.B. Fialho, and S.F. Pascholati. 2017. Potential of fumigation of orange fruits with volatile organic compounds produced by Saccharomyces cerevisiae to control citrus black spot disease at postharvest. Biol. Control 108:77-82. doi:10.1016/j.biocontrol.2017.02.009

Torres, R., C. Nunes, J.M. García, M. Abadias, I. Viñas, T. Manso, et al. 2007. Application of Pantoea agglomerans CPA-2 in combination with heated sodium bicarbonate solutions to control the major postharvest diseases affecting citrus fruit at several mediterranean locations. Eur. J. Plant Pathol. 118:73-83. doi:10.1007/s10658-007-9120-1

Torres, R., C. Solsona, I. Viñas, J. Usall, P. Plaza, and N. Teixidó. 2014. Optimization of packaging and storage conditions of a freezedried Pantoea agglomerans formulation for controlling postharvest diseases in fruit. J. Appl. Microbiol. 117:173-184. doi:10.1111/ jam.12511

Usall, J., J. Smilanick, L. Palou, N. Denis-Arrue, N. Teixidó, R. Torres, et al. 2008. Preventive and curative activity of combined treatments of sodium carbonates and Pantoea agglomerans CPA-2 to control postharvest green mold of citrus fruit. Postharvest Biol. Tec. 50:1-7. doi:10.1016/j.postharvbio.2008.03.001 
Wang, X., G. Chen, F. Huang, J. Zhang, K.D. Hide, and H. Li. 2012. Phyllosticta species associated with citrus diseases in China. Fungal Divers. 52(1):209-224. doi:10.1007/s13225-011-0140-y

Yun, Z., H. Gao, P. Liu, S. Liu, T. Luo, S. Jin et al. 2013. Comparative proteomic and metabolomic profiling of citrus fruit with enhancement of disease resistance by postharvest heat treatment. BMC Plant Biol. 13(44):1-16. doi:10.1186/1471-2229-13-44

Ze, Y., G. Huijun, L. Ping, L. Shuzhen, L. Tao, J. Shuai, et al. 2013. Comparative proteomic and metabolomic profiling of citrus fruit with enhancement of disease resistance by postharvest heat treatment. BMC Plant Biol. 13:1-16. doi:10.1186/1471-2229-13-44

Zhang, H., L. Wang, X. Zheng, and Y. Dong. 2007. Effect of yeast antagonist in combination with heat treatment on postharvest blue mold decay and Rhizopus decay of peaches. Int. J. Food Microbiol. 115:53-58. doi:10.1016/j.ijfoodmicro.2006.10.002
Zhou, Y., L. Deng, and K. Zeng. 2014a. Enhancement of biocontrol efficacy of Pichia membranaefaciens by hot water treatment in postharvest diseases of citrus fruit. Crop Prot. 63:89-96. doi:10.1016/j.cropro.2014.05.015

Zhou, Y., J. Ming, L. Deng, and K. Zeng. 2014b. Effect of Pichia membranaefaciens in combination with salicylic acid on postharvest blue and green mold decay in citrus fruits. Biol. Control 74:21-29. doi:10.1016/j.biocontrol.2014.03.007

Zhou, Y., L. Zhang, and K. Zeng. 2016. Efficacy of Pichia membranaefaciens combined with chitosan against Colletotrichum gloeosporioides in citrus fruits and possible modes of action. Biol. Control 96:39-47. doi:10.1016/j.biocontrol.2016.02.001 